\title{
A $220 \mathrm{GHz}$ 3D imaging radar with sub-cm ${ }^{3}$ voxel resolution for security applications
}

\author{
D.A. Robertson, D.G. Macfarlane \\ School of Physics \& Astronomy \\ University of St Andrews \\ St Andrews, Scotland \\ $\underline{\text { dar@st-and.ac.uk }}$
}

\author{
T. Bryllert \\ Wasa Millimetre Wave AB \\ c/o Chalmers University of Technology \\ Gothenburg, Sweden
}

\begin{abstract}
Radars operating at high millimetre and submillimetre wave frequencies are promising candidates for security applications such as people screening since they offer the possibility to form 3D images through clothing with sufficient resolution to detect concealed objects. High spatial resolution of order $1 \mathrm{~cm}$ can be achieved using practically sized antennas and high range resolution can be achieved using wideband FMCW chirps, e.g. $30 \mathrm{GHz}$, to yield $0.5 \mathrm{~cm}$ range bins. We present a 220 GHz test-bed 'Pathfinder' radar which achieves sub-cm ${ }^{3}$ voxel resolution with very high signal fidelity. The radar is used to derisk technology under development for next generation people screening systems.
\end{abstract}

Keywords- Millimetre wave radar, security, FMCW, 3D imaging, high resolution

\section{INTRODUCTION}

Over the last decade millimetre wave security scanners have emerged as the dominant technology for passenger body screening and have become a near ubiquitous presence at major international airport hubs around the world [1]. Despite this commercial success, the requirement for security imaging technology that facilitates faster passenger throughput at screening locations remains a highly desirable goal.

Submillimetre wave 3D imaging radar with high $\left(\sim \mathrm{cm}^{3}\right)$ voxel resolution offers the potential to resolve anomalous objects concealed under clothing. By using 3D shape information, intensity fluctuations caused by clothing and specular reflections are suppressed [2]. However, achieving high enough frame rates to yield reduced screening times remains a significant challenge for this technology.

The EU FP7 CONSORTIS (Concealed Object Stand-Off Real-Time Imaging for Security) project [3] aims to produce high resolution, real time security imaging with significantly improved throughput rates, and will combine a dual-band passive submillimetre wave imager and a $340 \mathrm{GHz}$ 3D imaging radar. In order to de-risk critical technology and provide representative phenomenological data, St Andrews and Wasa have constructed a $220 \mathrm{GHz}$ 'Pathfinder' radar imager to underpin the development of the future CONSORTIS radar.

\section{HARDWARE DESCRIPTION}

The 'Pathfinder' laboratory test-bed radar is a single-pixel imager based on a frequency multiplied transceiver and two-

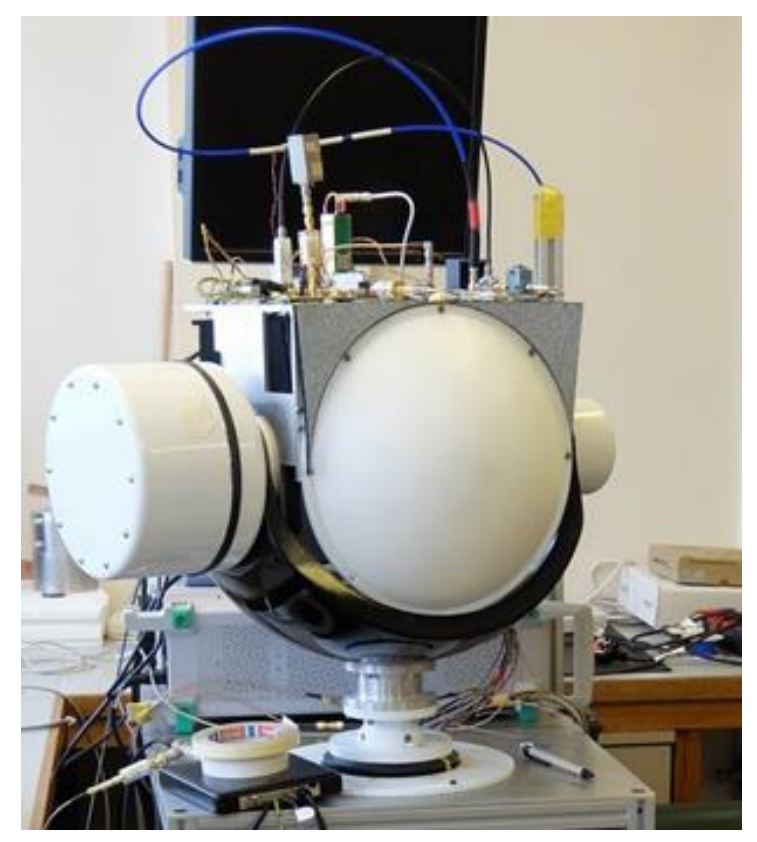

Fig. 1. $220 \mathrm{GHz}$ test-bed radar mounted on two-axis gimbal.

axis gimbal plus PC-based data acquisition. Whilst slow to acquire imagery the purpose of the radar is to prove the wideband chirp generator and transceiver technology and to gather data with a sub- $\mathrm{cm}^{3}$ voxel resolution to support the development of automatic image processing algorithms. The radar is shown in Fig. 1.

\section{A. Chirp Generator}

The chirp generator has been designed specifically to achieve the required wide bandwidth performance for this radar. For a $30 \mathrm{GHz}$ output chirp bandwidth, the chirp generator bandwidth is $1.25 \mathrm{GHz}$ at $8.958 \mathrm{GHz}$ i.e. $14 \%$. Chirps are generated with a direct digital synthesis (DDS) chip clocked at $3.5 \mathrm{GHz}$ which is upconverted onto a low phase noise stable local oscillator (STALO) to achieve high linearity, low phase noise, high speed, wideband chirps [4]. The phase noise of the chirp generator is dominated by that of the STALO, which in the experiments described below was an Anritsu MG3692C synthesiser. The chirp generator output power is $+7 \pm 2 \mathrm{dBm}$, sufficient to drive the transceiver. 


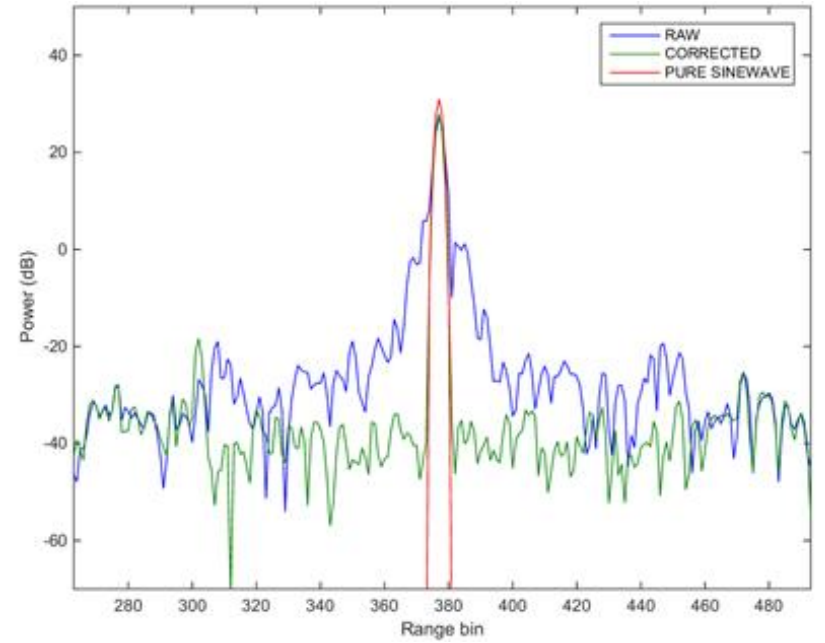

Fig 2. Raw and corrected range point responses compared with pure sinewave.
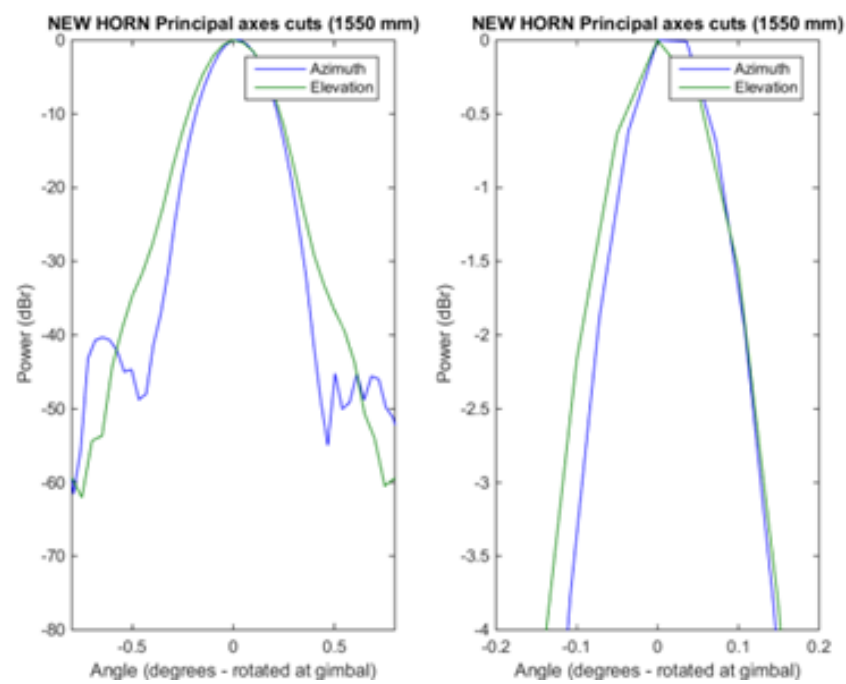

Fig 4. Two-way (radar) beam pattern principal planes at focal plane.

\section{B. Transceiver}

The transceiver uses $\mathrm{x} 24$ frequency multiplication in the sequence x4 MMIC - x3 Schottky - x2 Schottky. The final stage is configured as a self-mixing, balanced diode multiplier thus acting as the final transmit doubler and as a sub-harmonic mixer on receive [5]. The transceiver output power varies between +2 and $+5 \mathrm{dBm}$ over 200 to $230 \mathrm{GHz}$. The receive conversion loss is $-17 \mathrm{~dB}$.

\section{Optics}

The transceiver is coupled to free-space with a smoothwalled conical feedhorn, which points vertically downwards. A low return loss feedhorn is essential to ensure the correct operation of the waveguide hybrid at the front end of the selfmixing multiplier. A plane mirror at $45^{\circ}$ deflects the beam forwards where it is focussed by a plano-convex lens made of HDPE. The lens focusses the beam to a tight spot a few metres from the radar. The lens design was optimised to achieve a symmetric beam with low sidelobes and a well-controlled

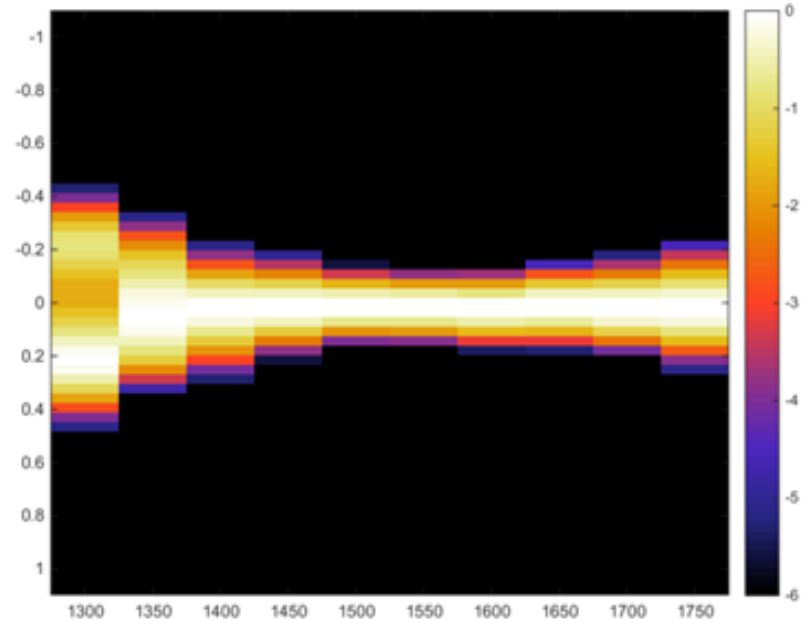

Fig 3. Beam diffraction showing focal plane is at $1.55 \mathrm{~m}$.

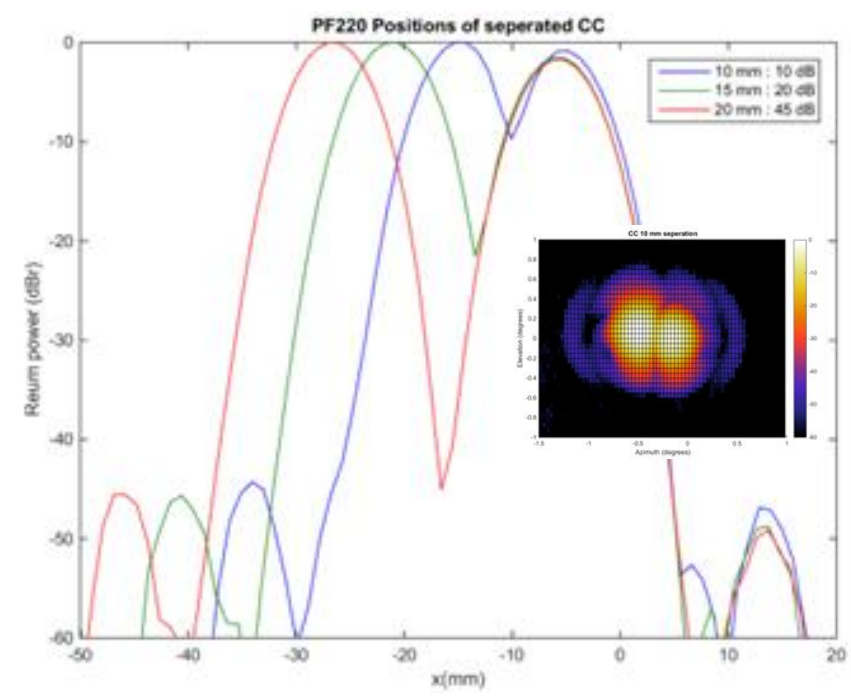

Fig 5. Two-target separation test for 20, 15 and $10 \mathrm{~mm}$ separations plus radar image of two point targets separated by $10 \mathrm{~mm}$ (inset).

depth of field to maximise the radar image quality. Sidelobe level is principally a function of the illuminating feedhorn. The surfaces of the lens are not anti-reflection treated. The whole assembly is mounted on a two-axis elevation-over-azimuth gimbal with the pivot axis slightly behind the lens.

\section{RANGE PROFILE PERFORMANCE}

Wideband sub-millimetre wave radars based on frequency multiplication chains tend to exhibit quite badly degraded raw point responses $[2,6]$. The range profile performance of the radar was characterised by measuring the point response obtained from a sub-beamwidth target placed at the beam focus. In contrast to these other groups, the raw point response of our radar is extremely clean given the large fractional bandwidth. This is attributed to a combination of the high linearity and good amplitude flatness of the chirp generator and the well-controlled group delay and good power flatness of the transceiver multiplier chain. As is the established practice for such wideband high frequency radars, residual nonlinearities in the transmitted chirp can be compensated for by calibrating the 

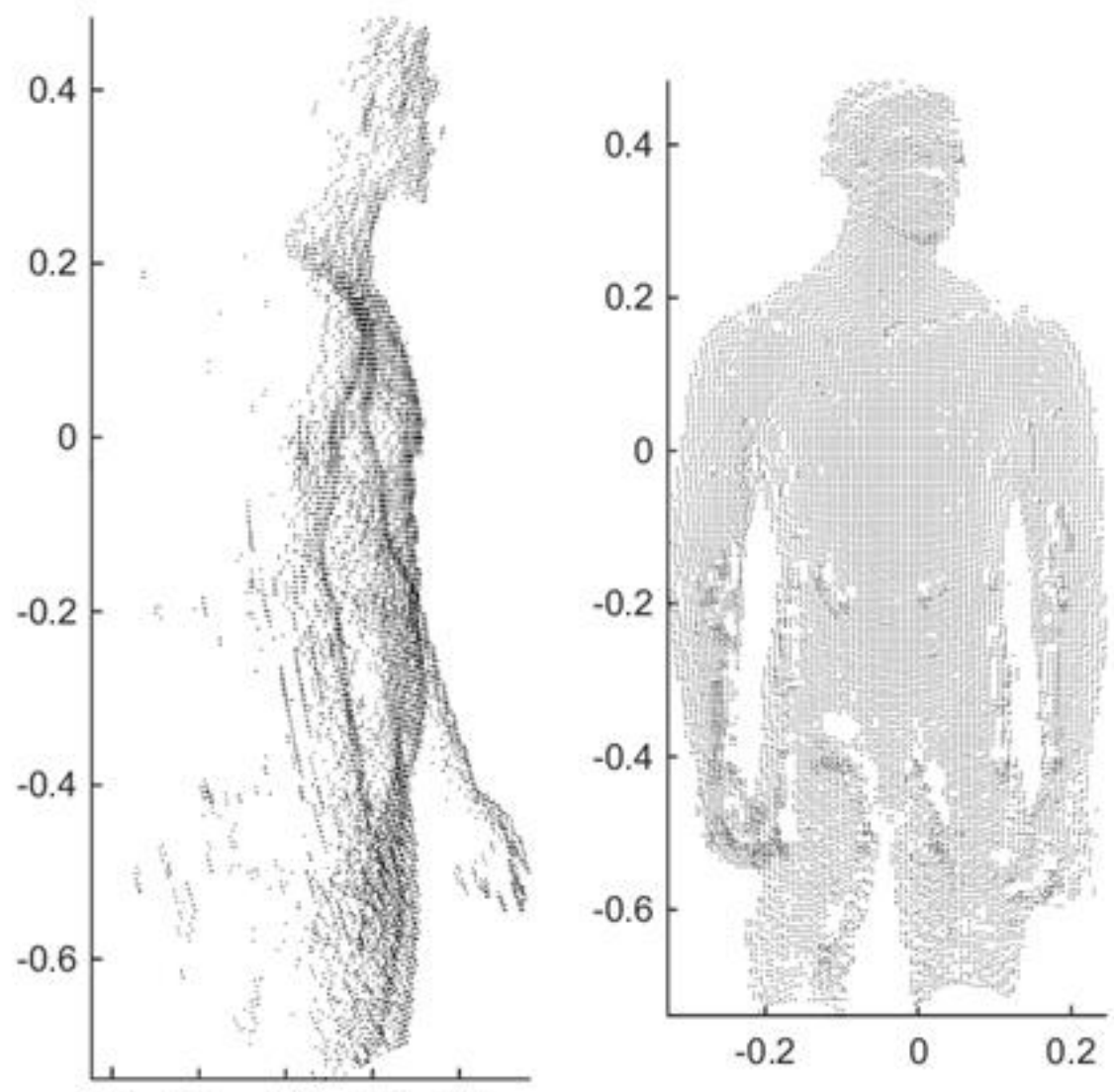

\section{P220 POINT CLOUD xyz offset (m)}

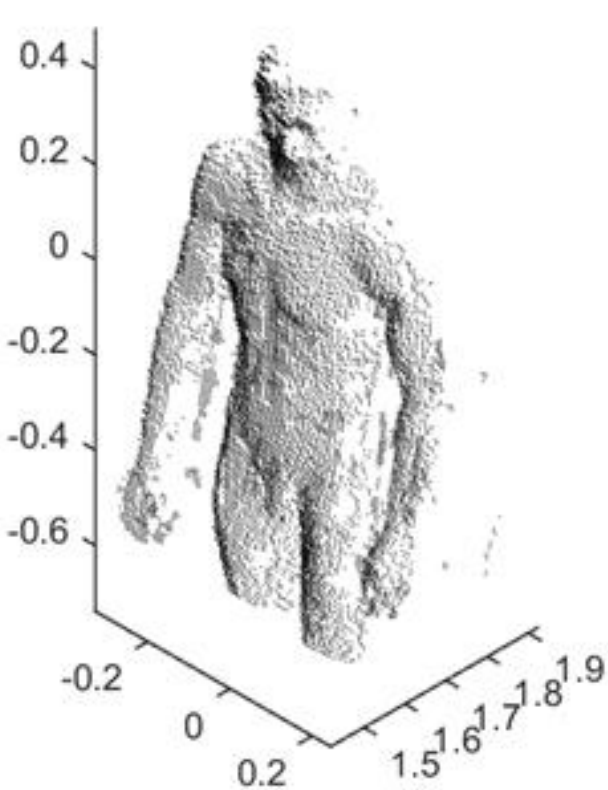

$\begin{array}{lllll}1.9 & 1.8 & 1.7 & 1.6 & 1.5\end{array}$

Fig 6. Side, front and isometric views of 3D point cloud of mannequin acquired with $220 \mathrm{GHz}$ radar.

point response from a reference target at a specific range and normalizing the received radar spectrum [2]. Whilst the calibration is strictly only valid at that range, it is effective over a reasonable range swath, and at least over the depth of field of the beam. Fig. 2 shows the raw and calibrated point response of the radar compared with that of a pure, numerical sinewave. It can clearly be seen that the corrected point response follows the theoretical resolution limit over a dynamic range of $>60$ $\mathrm{dB}$.

\section{OPTICAL PERFORMANCE}

The focussing characteristics of the radar beam were characterised by imaging a small sub-beamwidth target positioned at different distances. The resulting series of beam profiles is shown in Fig. 3 and reveals the plane of focus lies at a range of $1.55 \mathrm{~m}$ from the front of the radar.

The beam quality and spatial resolution at the focal plane are excellent. Fig. 4 shows the good beam symmetry and a two-way (radar) $3 \mathrm{~dB}$ width of $\pm 0.1^{\circ}$ which corresponds to a spot size of $<1 \mathrm{~cm}$ when accounting for the gimbal geometry. The sidelobe level of $-40 \mathrm{~dB}$ (two-way) is consistent with the sidelobes expected for the lens design when fed by the smoothwalled conical feedhorn.

The excellent spatial resolution was confirmed by imaging two closely-spaced sub-beamwidth targets as they are brought closer together. Fig. 5 shows lateral beam profiles for separation of 20,15 and $10 \mathrm{~mm}$. Note that even at $10 \mathrm{~mm}$ separation there is still a $10 \mathrm{~dB}$ dip between the peaks.

\section{3D IMAGING RESULTS}

The radar has been used to collect 3D imagery from a lifesize mannequin and various test targets. Data acquisition is fully automated using a laptop which performs the gimbal positioning, IF output sampling and FMCW processing including range calibration. Point cloud extraction from the raw 3D data set is performed offline in MATLAB. Typical chirp settings are $30 \mathrm{GHz}$ bandwidth and $100 \mu$ s duration. Image acquisition is slow due to limitations of the data capture card so only static targets can be used. For example, a 0.6 x $0.6 \mathrm{~m}$ field of view image with $1 \mathrm{~m}$ range swath takes 90 minutes to acquire.

Fig. 6 shows side, front and isometric views of a 3D point cloud of the mannequin. The benefits of sub- $\mathrm{cm}^{3}$ voxel resolution are evident as the fine details in the surface profile of the mannequin which are well resolved.

\section{CONCLUSIONS}

We have presented the 'Pathfinder' $220 \mathrm{GHz}$ test-bed radar capable of sub- $\mathrm{cm}^{3}$ voxel resolution $3 \mathrm{D}$ imaging which demonstrates the high performance achievable with a wideband DDS-based chirp generator and self-mixing multiplier based transceiver. The raw point response of the radar is extremely 
good and with minor calibration the range resolution is transform-limited over a $>60 \mathrm{~dB}$ dynamic range. The test lens has demonstrated $<1 \mathrm{~cm}$ spatial resolution enabling the clear distinction between point targets spaced as close as $1 \mathrm{~cm}$.

The 'Pathfinder' radar de-risks critical technology for the CONSORTIS $340 \mathrm{GHz}$ 3D imaging radar which is currently under development. That radar will acquire and process 3D imagery of the same high volumetric resolution as achieved with 'Pathfinder' but at a $10 \mathrm{~Hz}$ frame rate, suitable for high throughout security screening.

\section{ACKNOWLEDGMENT}

The authors thank Dr Ewan Finlay of InDepth Optics Ltd. for Zemax lens design and the University's Mechanical Workshop for fabricating the hardware.

\section{REFERENCES}

[1] Schouten, P "Security as controversy: Reassembling security at Amsterdam Airport”, Security Dialogue, vol. 45, February 2014, pp2342, doi:10.1177/0967010613515014

[2] K.B. Cooper, et al., "THz Imaging Radar for Standoff Personnel Screening," IEEE Trans. Terahertz Science and Technology, Vol. 1, No. 1, 2011, pp. 169 - 182

[3] CONSORTIS website: www.consortis.eu

[4] D. A. Robertson, S. L. Cassidy, and D. R. Bolton, "Nonlinearity and phase noise effects in $340 \mathrm{GHz}$ 3D imaging radar", Proc. SPIE 8715, Passive and Active Millimeter-Wave Imaging XVI, 87150M (31 May 2013); doi: 10.1117/12.2015774.

[5] T. Bryllert, V. Drakinskiy, K. B. Cooper, and J. Stake, "Integrated 200 240-GHz FMCW Radar Transceiver Module”, IEEE Trans. Microwave Theory Tech., Vol. 61, No. 10, 2013, pp. 3808 - 3815.

[6] Grajal, J., Badolato, A., Rubio-Cidre, G., Ubeda-Medina, L., Mencia-Oliva, B., Garcia-Pino, A., Gonzalez-Valdes, B., and Rubinos, O, " 3-D High-Resolution Imaging Radar at $300 \mathrm{GHz}$ With Enhanced FoV", IEEE Trans. Microwave Theory Tech., Vol. 63, No. 3, 2015, pp. 1097-1107. 